\title{
A Closed Form Solution of Convective Mass Transfer Model for Intracellular Calcium Response of Endothelial Cells
}

\author{
VINEET KUMAR ${ }^{a, \dagger}$, R.N. PANDEY ${ }^{b}$ and S.N. UPADHYAY ${ }^{*} *$ \\ ${ }^{a}$ Department of Chemical Engineering \& Technology, ${ }^{b}$ Department of Applied \\ Mathematics, Institute of Technology, Banaras Hindu University, \\ Varanasi - 221005, India
}

(Received 7 November 1997; Revised 26 June 1998)

Endothelial cells, lining the entire vascular system, respond to change in concentration of specific agonist like adenosine triphosphate (ATP) by increasing cytosolic $\mathrm{Ca}^{2+}$ concentration, producing prostacyclin, endothelium derived relaxing factor and ectoenzymes. Three different ecto-enzymes metabolize ATP in three steps (ATP $\rightarrow$ $\mathrm{ADP} \rightarrow \mathrm{AMP} \rightarrow$ adenosine). Normally experiments with endothelium are carried out in a rectangular flow chamber provided with a cell surface at one of its walls and feed stream containing ATP. The ATP concentration near the cell surface depends upon two factors, rate of its degradation and the rate at which it reaches from upstream. Closed form solutions for the concentration profile of ATP in such a flow chamber indicates that concentration near the cell surface is lower than the bulk concentration depending on the activity of ecto-enzymes and it increases with increase in tangential flow rate (shear stress). This indicates that shear induced response of endothelial cell (at least for low shear rate) may be due to change in ATP concentration near the cell surface which is sensed by purinoreceptors instead of a mechanoreceptor. Several workers have tried to investigate this problem analytically. Unfortunately, solutions obtained by these workers have limited success. In the present work, exact solution of the problem has been obtained in terms of a confluent hypergeometric function. Solution of the transformed equation gives accurate results even in the entrance region of the flow chamber which eliminates the need of solutions based on approximate methods like perturbation or finite difference techniques.

Keywords: Endothelium; ATP; Shear stress; Calcium response; Mass transfer

\footnotetext{
* Corresponding author. Tel.: 91542317192 . Fax: 91542316925.

${ }^{\dagger}$ Presently at School of Biochemical Engineering.
} 


\section{INTRODUCTION}

A continuous monolayer of endothelial cells lines the entire vascular system and forms the interface between the blood and the tissue. This layer is responsible for regulating several crucial physiological processes like maintenance of vascular tone and modulation of platelet adhesion. It also acts like a permeability barrier and modulates the exchange of nutrients and metabolic end-products between blood and neighboring cells through the adjacent vessel wall [1-5] and production of vasoactive compounds such as prostacyclin and endothelium derived relaxing factor (EDRF) [6] in response to specific agonist such as histamine [7], thrombin [8] and adenosine-triphosphate (ATP) $[9,10]$.

In spite of concerted attempts by several investigators [11-23] to study the effects of shear stress and agonist concentration on the extraand intracellular changes, both theoretically and experimentally, the mechanism involved in these processes is still rather poorly understood. However, most of the investigators suggest two separate mechanisms for processes occurring near the endothelial cell surface. First, the shear stress acting on the endothelial cell surface directly affects its signal-transduction system which starts all types of cellular changes. Increase in cytosolic $\mathrm{Ca}^{2+}$ concentration in the endothelial cell by increasing shear rate has been inferred by a several-fold increase in prostacyclin production [13-15] and dilation of blood vessels [24-26]. These changes in endothelial cell activity, however, were not shown to be graded with respect to shear stress [12].

The second mechanism, which has been overlooked by several investigators, suggests that the change in cell activity is due to the change in mass transfer rate and concentration of agonist and metabolite near the cell surface which depend solely on the hemodynamics and enzyme activity. Production of prostacyclin and endothelium derived relaxing factor is due to the presence of specific agonists like thrombin, histamine [6-8] and ATP [9-11]. Metabolism at the endothelial cell surface also affects the mass transport of agonists. For regulating the near wall concentration of vasoactive agents, endothelial cells posses several membrane bound ecto-enzymes that degrade vasoactive substances [27]. For example bradykinin is broken into an inactive peptide through the action of angiotensin-converting enzymes [28]. Similarly adenine nucleotides, which are important modulators of 
both vascular tone [29] and thrombotic cascade [30], are inactivated through the successive dephosphorylation of ATP to adenosine diphosphate (ADP) and then to adenosine monophosphate (AMP) and finally to adenosine by three separate ecto-enzymes. Binding of extracellular ATP to a purinoreceptor $\left(\mathrm{P}_{2 \mathrm{y}}\right)$ on the cell surface starts this endothelial cell metabolism $[9,10]$. A sequence of events leading to a rapid increase in the cytosolic $\mathrm{Ca}^{2+}$ concentration are initiated due to this action. $\mathrm{Ca}^{2+}$ acts as second messenger and causes activation of the metabolic pathways resulting in the production of a vasoactive factor [31-33]. The circulating level of ATP is reduced by the activity of ecto-enzymes on the exterior surface of the endothelial cell plasma membrane. If the activity of these enzymes are sufficiently high, the ATP concentration near the cell surface can be sufficiently lower than the bulk concentration. Thus the ectonucleotidases may contribute to the development of an ATP depleted zone within the flow established boundary layer that limits agonist availability to the $\mathrm{P}_{2 \mathrm{y}}$-purinoreceptor.

To test the validity of this newly emerging mass transfer based model, several workers [11-13], have tried to investigate the problem experimentally in rectangular channels with cultured endothelial cells as shown in Fig. 1. The hydrolyzable form of ATP was used in hydroxyethylpiprazine-N'-2-ethanesulfonic acid (HEPES) buffer saline solution as the profusing medium. Analytical solution for concentration profile in rectangular channel using power series method for a similar problem has been obtained by Nollert and McIntire [11], Grimsrud and Babb [34] and Colton et al. [35] by evaluating three, one and seven eigenvalues, respectively. Obviously, these workers had only limited success in solving the problem (particularly for small channel length). Moreover, three separate approaches (power series solution, perturbation solution and finite difference solution) were required to cover the entire length [11]. In the present work the problem has been solved by using the fast converging confluent hypergeometric function (Kummer's function). Due to converging nature of the function, it is possible to handle a large number of eigenvalues which give satisfactory result even near the entrance of the channel (about $1 \mathrm{~cm}$ from the entrance). Availability of large number of eigenvalues eliminates the need of any approximate solution based on perturbation, finite element or finite difference techniques. 


\section{MATHEMATICAL FORMULATION}

Figure 1 shows the schematic diagram of a rectangular flow chamber having one reacting wall (at $y=h$ ) made of endothelial cells (similar to those used by experimental investigators). Fluid containing ATP with the inlet concentration $C_{0}$ enters the chamber through the left face. As shown in the figure, for laminar flow, there is no fluid flow near the wall and delivery of ATP to the purinoreceptor $\left(\mathrm{P}_{2 \mathrm{y}}\right)$ is controlled solely by molecular diffusion. Degradation of ATP reduces its concentration and establishes a concentration gradient near the cell surface. However, the concentration gradient of ATP near the cell surface depends on flow rate. At low flow rates, the degradation of ATP by the ecto-enzymes exceeds its delivery by diffusion, hence the steady state concentration of ATP near the endothelial cell surface is low. At high flow rates, however, convection enhances the delivery of ATP in the bulk from upstream, then the diffusion of ATP from bulk to surface will exceed the rate of its degradation by the ectonucleotidase, causing higher concentration of ATP at the cell surface. A diagram of

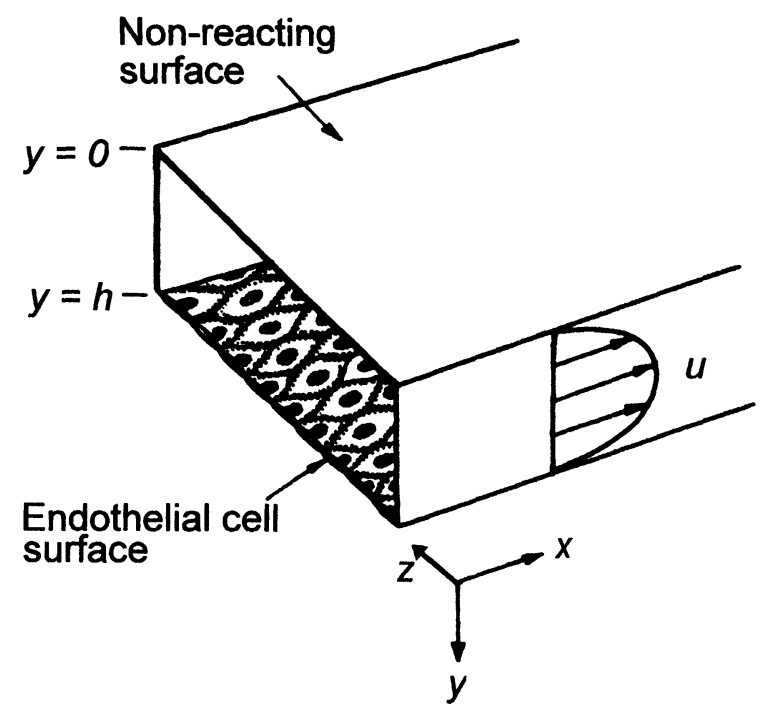

FIGURE 1 Diagram showing parallel plate geometry of the flow chamber with parabolic velocity profile and orientation of co-ordinate axes. 


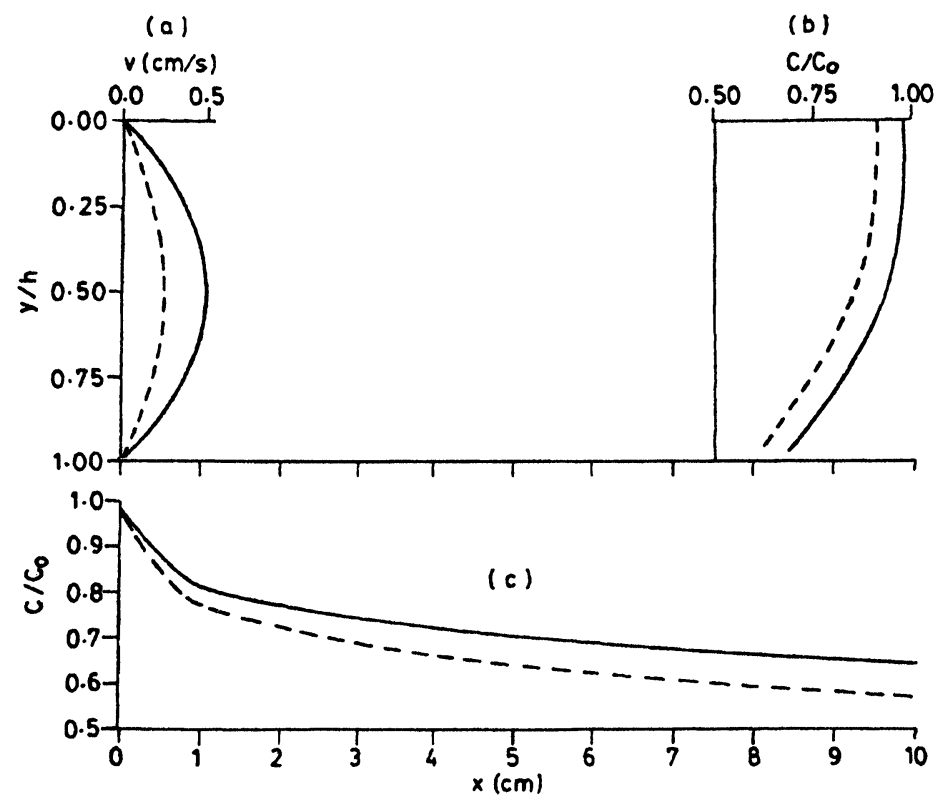

FIGURE 2 Schematic diagram showing two velocity profiles (a), corresponding dimensionless concentration profiles in the bulk of liquid (b) and near the endothelial cell surface (c).

velocity and concentration profiles at two different flow rates are shown in Fig. 2.

With this in view, in the present work, two dimensional laminar flow is being considered in a thin channel having rectangular cross-sectional area. Conversion of the reactant (ATP) into an inert substance has been reported to follow a first order chemical reaction $[10,11,36]$ that takes place at the surface of one wall of the channel (at $y=h)$. Further, it is assumed that the uniform inlet concentration of ATP (at $x=0$ ) is $C_{0}$ and diffusive mass transfer is negligible in the direction parallel to the wall of the channel and that there is no velocity component in $y$ direction $\left(v_{y}=0\right)$.

The velocity profile in rectangular channel has been developed by Berman [37], Kozinski et al. [38] and Kleinstreuer and Paller [39] for different physical conditions, i.e., for impermeable wall, permeable wall at both sides and at single side of the channel, respectively. However, with minor modification and rearrangement one may obtain the 
following velocity profile in an impermeable rectangular channel

$$
v_{x}=6\langle u\rangle\left\{\frac{y}{h}-\left(\frac{y}{h}\right)^{2}\right\},
$$

where $\langle u\rangle$ is the average velocity of the fluid flowing in the channel. With these assumptions, the equation of continuity for constant density and diffusivity under steady state conditions reduces to:

$$
6\langle u\rangle\left\{\frac{y}{h}-\left(\frac{y}{h}\right)^{2}\right\} \frac{\partial C}{\partial x}=D \frac{\partial^{2} C}{\partial y^{2}} .
$$

From the definition of the problem, one wall of the rectangular channel (at $y=h$ ) provides a reacting surface with first order reaction, hence, at the steady state, the mass flux due to diffusion is equal to the mass consumed at the surface in chemical reaction. Thus

$$
-D \frac{\partial C}{\partial y}=k C,
$$

where $k(\mathrm{~m} / \mathrm{s})$ is the first order rate constant based on unit surface area. This gives

B.C. I:

$$
\left.\frac{\partial C}{\partial y}\right|_{y=h}=-\frac{k C}{D} \quad(\text { for all } x) .
$$

Also, another wall (at $y=0$ ) is non-reacting which leads to B.C. II:

$$
\left.\frac{\partial C}{\partial y}\right|_{y=0}=0 \quad(\text { for all } x)
$$

and a uniform concentration at inlet gives

B.C. III:

$$
C=C_{0} \quad \text { at } x=0(\text { for all } y) .
$$

For simplicity, dimensionless variables may now be defined as:

$$
\begin{gathered}
c=C / C_{0}, \\
\gamma=y / h,
\end{gathered}
$$


and

$$
\xi=x D /\langle u\rangle h^{2}
$$

which reduces Eq. (2) to

$$
6\left(\gamma-\gamma^{2}\right) \frac{\partial c}{\partial \xi}=\frac{\partial^{2} c}{\partial \gamma^{2}}
$$

and the boundary conditions become

B.C. I:

$$
\left.\frac{\partial c}{\partial \gamma}\right|_{\gamma=1}=-\frac{k h c}{D}=-\phi^{2} c \quad(\text { for all } \xi)
$$

B.C. II:

$$
\left.\frac{\partial c}{\partial \gamma}\right|_{\gamma=0}=0 \quad(\text { for all } \xi)
$$

B.C. III:

$$
c=1 \quad \text { at } \xi=0(\text { for all } \gamma) .
$$

The term $k h / D$ in Eq. (7a) is the Thiele modulus $\left(\phi^{2}\right)$ based on a first order surface reaction.

\section{SOLUTION OF THE PROBLEM}

\section{Concentration Profile}

By separation of variables and two transformations (see Appendix), the complete solution of Eq. (6) and associated boundary conditions becomes

$$
\begin{aligned}
c= & \sum_{n=0}^{\infty} A_{n} \exp \left(-\lambda_{n}^{2} \xi / 6-z_{n} / 2\right)\left[M\left(a_{n}, \frac{1}{2}, z_{n}\right)\right. \\
& \left.-\sqrt{z_{n}} \alpha_{n} / \beta_{n} M\left(a_{n}+\frac{1}{2}, \frac{3}{2}, z_{n}\right)\right]
\end{aligned}
$$


where $\lambda_{n}$ is the $n$th eigenvalue, $M$ is the confluent hypergeometric function (Kummer's function),

$$
\begin{gathered}
z_{n}=\lambda_{n}\left(\gamma-\frac{1}{2}\right)^{2}, \\
a_{n}=\frac{1}{4}-\lambda_{n} / 16, \\
\alpha_{n}=2 a_{n} M\left(a_{n}+1, \frac{3}{2}, \lambda_{n} / 4\right)-\frac{1}{2} M\left(a_{n}, \frac{1}{2}, \lambda_{n} / 4\right)
\end{gathered}
$$

and

$$
\begin{aligned}
\beta_{n}= & \left\{\frac{\lambda_{n}}{\sqrt{\lambda_{n}}}-\frac{\sqrt{\lambda_{n}}}{2}\left(\phi^{2}-\frac{\lambda_{n}}{2}\right)\right\} M\left(a_{n}+\frac{1}{2}, \frac{3}{2}, \frac{\lambda_{n}}{4}\right) \\
& +\frac{\lambda_{n}^{3 / 2}\left(2 a_{n}+1\right)}{6} M\left(a_{n}+\frac{3}{2}, \frac{5}{2}, \frac{\lambda_{n}}{4}\right) .
\end{aligned}
$$

The infinite series obtained in Eq. (8) converges faster than the series obtained by Nollert and McIntire [11]. Thus the concentration of ATP in the flow chamber could be calculated easily and more accurately. Also, due to the fast converging nature of Kummer's function, it has become possible to calculate much more eigenvalues. Having sufficiently large number of eigenvalues, it is possible to calculate concentration distribution even for $\xi=1 \times 10^{-6}$ with an error less than $1 \%$. Here, it is worth mentioning that the result at small $\xi$ is valid only if velocity profile is fully developed and the value of $\phi^{2}$ is very small.

\section{Shear Stress}

To predict wall concentration as a function of shear stress, one may express $\xi$ in terms of $\tau$. From Eq. (1) we have

$$
\frac{\mathrm{d} v_{x}}{\mathrm{~d} y}=6\langle u\rangle\left(1 / h-2 y / h^{2}\right) .
$$

Near the endothelial cell surface $(y=h)$, assuming Newtonian behavior of the fluid (i.e., HEPES), shear stress may be calculated by

$$
\tau=-\left.\mu \frac{\mathrm{d} v_{x}}{\mathrm{~d} y}\right|_{y=h}
$$


or

$$
\tau=6 \mu\langle u\rangle / h .
$$

Substituting $\langle u\rangle$ from Eq. (11) in Eq. (5c) we get

$$
\xi=\frac{6 \mu x D}{\tau h^{3}} .
$$

Equation (8) along with Eq. (12) gives the concentration profile as a function of shear stress $(\tau)$ and dimensionless distance $(\gamma)$ at a particular value of $\xi$. These equations may be used to compare the predicted value of ATP concentration near the cell surface and experimentally observed endothelial cell response under identical conditions.

\section{DISCUSSION}

The accuracy of the results depend upon number of eigenvalues used. Grimsrud and Babb [34], Colton et al. [35] and Nollert and McIntire [11] tried to solve similar problems using one, seven and three eigenvalues, respectively. The actual number of eigenvalues required is, however, much higher. The contour plot (Fig. 3) shows the number of eigenvalues required to calculate wall concentration at a particular set of $\phi^{2}$ and $\xi$, within an error of less than $1 \%$. It is clear from this figure that more and more eigenvalues are required for small values of $\xi$ and large Thiele modulus.

On the basis of concentration profile obtained in the present work, it seems that one of the ways through which cells are capable of responding to shear stress is indirectly through the flow rate caused change in concentration of agonist near the cell surface which in turn, activates cells to respond accordingly.

The concentration profile in the parallel plate flow chamber is shown in Fig. 4 for $\phi^{2}=1.0$. The values obtained by present approach using Kummer's function are shown in continuous lines and those obtained by Nollert and McIntire [11] using series solution are shown as point values. The close agreement between the two solutions for $\xi>0.05$ is clearly evident, however, at $\xi=0.001$, there is a 


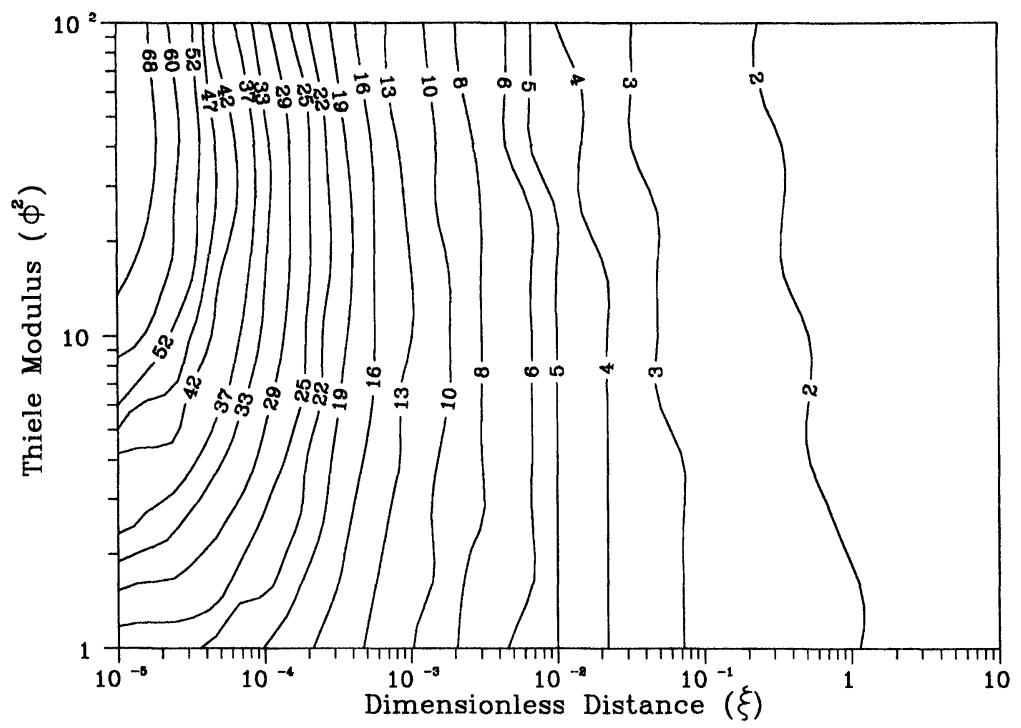

FIGURE 3 Minimum number of eigenvalues required to predict wall concentration with an error less than $1 \%$ for a given value of $\xi$ and $\phi^{2}$. Contour lines indicate left boundary of the region that requires a constant number of eigenvalues.

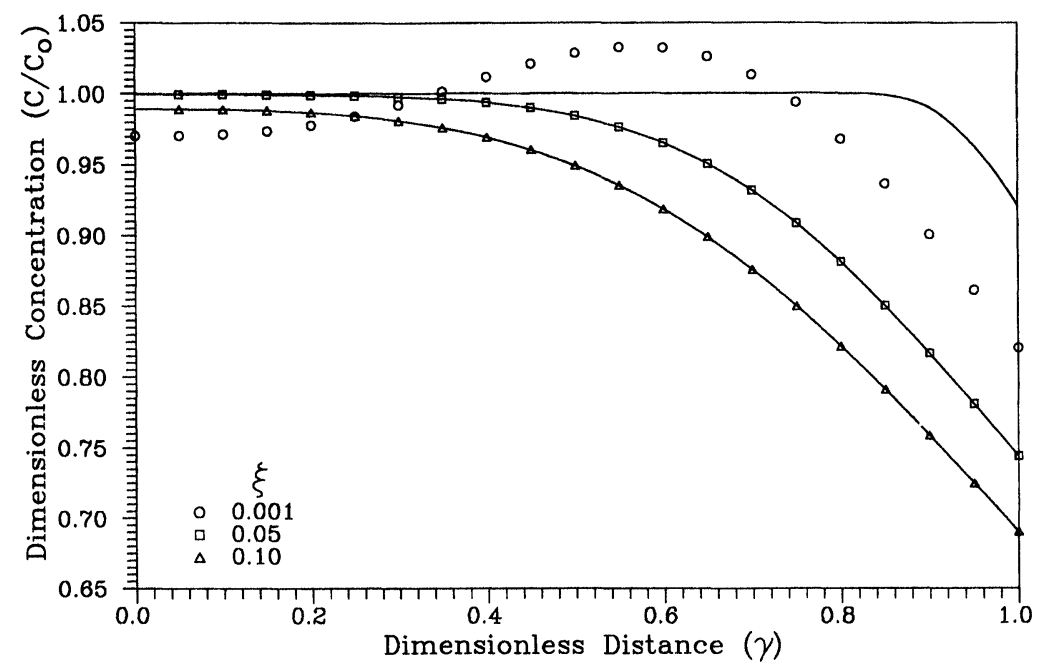

FIGURE 4 Comparison of the dimensionless concentration $\left(C / C_{0}\right)$ as a function of radial distance $(\gamma)$ in a rectangular channel obtained by power-series solution (point values) and solution based on Kummer's function (solid line) at $\phi^{2}=1.0$ and different values of dimensionless axial distances $(\xi)$. 
considerable deviation in the concentration profile. It is also clear from this figure that in the entrance region $(\xi<0.05)$ the bulk concentration is almost equal to the inlet concentration for more than about half the width of the flow chamber and there is a steep concentration change near the cell surface. Further down the length of the chamber, the concentration of ATP is much lower and consequently the concentration gradient near the wall is less pronounced. This indicates that less amount of ATP is approaching the surface by diffusion and hence less wall concentration at higher values of $\xi$ is observed.

Figure 5 shows the concentrations of ATP on the cell surface in a parallel plate flow chamber as a function of dimensionless axial position obtained by series and perturbation solutions [11], computer simulation [40] and the solution based on Kummer's function (present work) for $\phi^{2}=1$ and 4 . The series solution gives accurate values of concentration for $\xi>0.05$ whereas the perturbation solution gives accurate results for very small $\xi$ and $\phi^{2}$. Results of the computer simulation and the present solution closely correspond to the series solution and to the perturbation solution for large and small values of $\xi$,

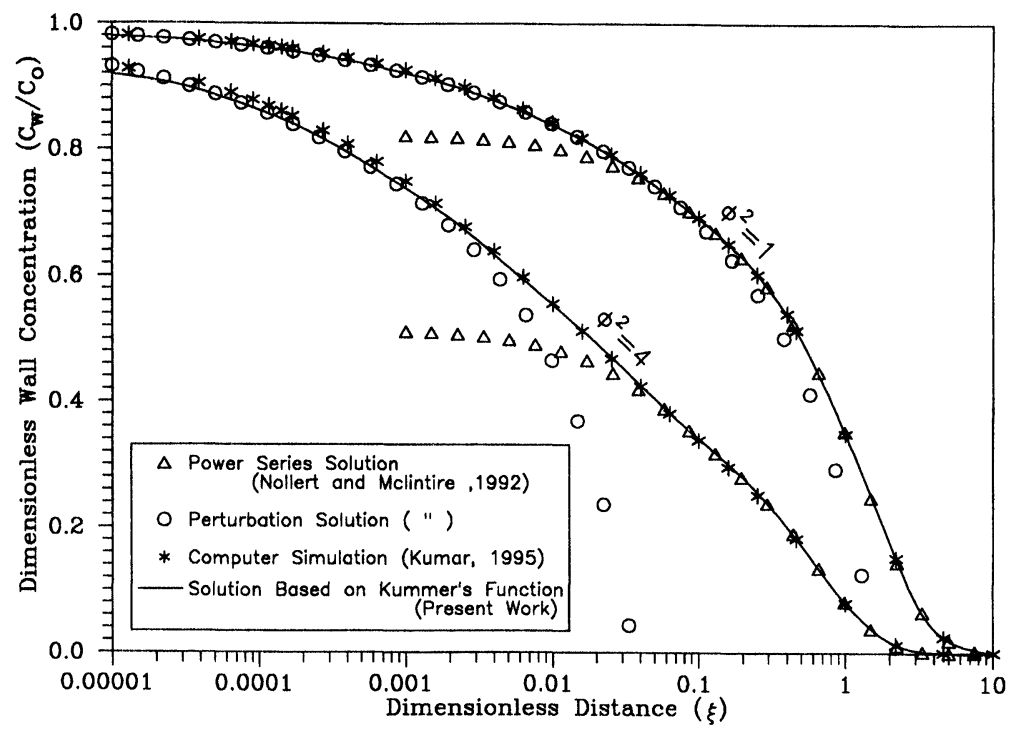

FIGURE 5 Variation of dimensionless wall concentration $\left(C_{\mathrm{W}} / C_{0}\right)$ with dimensionless axial distance $(\xi)$ in a rectangular channel calculated by four different approaches at $\phi^{2}=1$ and 4 . 
respectively. Although, computer simulator gives accurate results for the entire range of $\xi$ but it takes much more computation time (about $20 \mathrm{~min}$ on an Intel 80486 base PC at $66 \mathrm{MHz}$ ). On the other hand, computation time required for Kummer's function based solution, including evaluation of 75 eigenvalues, is about $1.5 \mathrm{~min}$ on the same PC.

Figure 6 shows the variation of dimensionless wall concentration with shear stress at a distance $2 \mathrm{~cm}$ from the leading edge of the flow chamber at $\phi^{2}=1$. To calculate shear stress, the channel geometry was considered to be that of Nollert and McIntire [11] and two experimentally determined values of steady state wall concentrations are also plotted as point values in this. To calculate steady state wall concentration it is assumed that the peak value of the fluorescence ratio is proportional to the inlet concentration of the feed containing ATP and the steady state fluorescence ratio is proportional to the steady state wall concentration. Even without knowing the value of the proportionality constant, dimensionless wall concentration can be

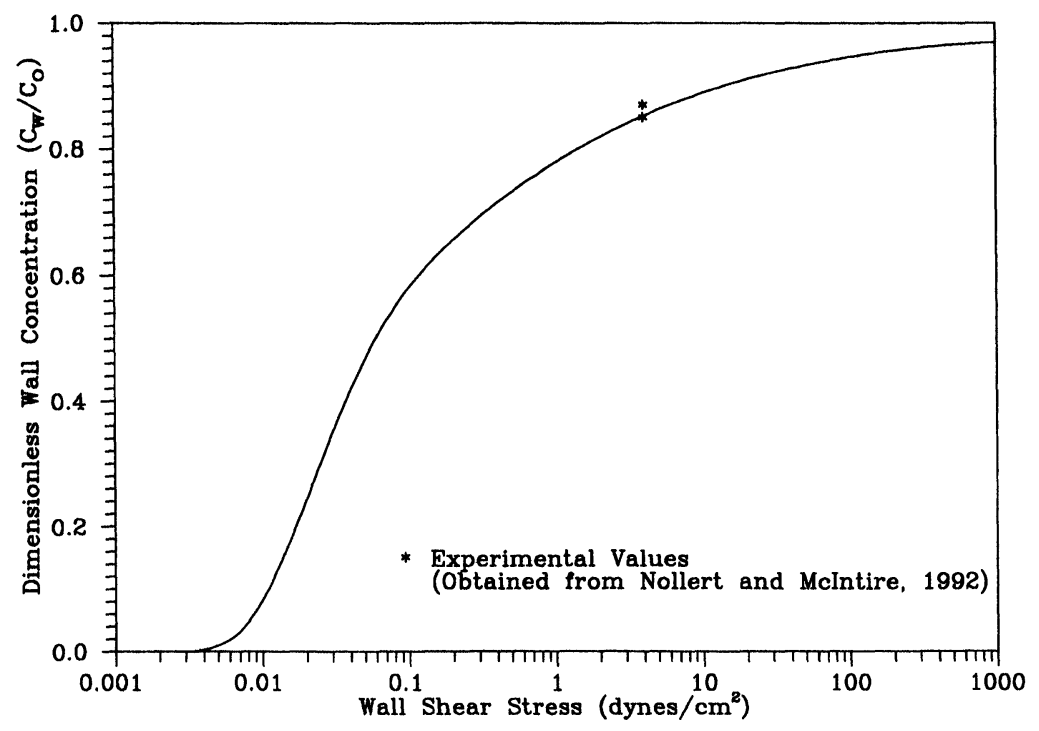

FIGURE 6 Comparison of dimensionless wall concentration predicted analytically (using Eqs. (8) and (12)) and those obtained experimentally by Nollert and McIntire [11] in a rectangular channel at a distance $2 \mathrm{~cm}$ from the inlet as a function of wall shear stress $\left(h=0.0218 \mathrm{~cm} ; D=2.6 \times 10^{-6} \mathrm{~cm}^{2} / \mathrm{s} ; \phi^{2}=1.0\right)$. 
calculated by taking the ratio of the two quantities. It is clear from this figure that the experimental values are in excellent agreement with the calculated values.

\section{CONCLUSIONS}

It seems, prior to the present work, no acceptable exact solution of the problem has been obtained. Present solution is applicable over a wide range of channel lengths compared to the range covered by previous workers. Thus it gives a more realistic picture of the phenomenon occurring near the cell surface. The exact solution obtained by Nollert and McIntire [11] is valid for $\xi>0.05$ (i.e., for a distance more than about $14 \mathrm{~cm}$ from the entrance, at the conditions specified for their experimental setup). On the basis of the present solution and those of earlier workers, it is observed that the agonist concentration at the endothelial cell surface increases with increase in tangential flow rate, hence there is a possibility that the endothelial cell response to the applied shear stress may be due to change in agonist concentration rather than the direct effect of shear stress.

There is evidence of increase in prostacyclin production by endothelial cell when shear stress is increased in absence of ATP [15]. However, the effect of agonist like ATP cannot be denied. Two experimental points obtained by Nollert and McIntire [11] are in excellent agreement with the calculated values assuming effect of ATP concentration alone. This shows that one of the possible mechanisms of vascular endothelial cell to detect changes in flow rate may be by the detection of change in concentration of agonist near the cell surface.

\section{NOMENCLATURE}

$\begin{array}{ll}A, B & \text { constant } \\ C & \text { concentration }(\mathrm{g} / \mathrm{ml}) \\ C_{0} & \text { feed concentration }(\mathrm{g} / \mathrm{ml}) \\ c & \text { concentration }\left[C / C_{0}\right](\text { dimensionless }) \\ D & \text { diffusivity }\left(\mathrm{cm}^{2} / \mathrm{s}\right) \\ h & \text { channel height }(\mathrm{cm})\end{array}$




$\begin{array}{ll}K_{1}, K_{2} & \begin{array}{l}\text { constant of integration } \\ \text { first order reaction rate constant based on unit sur- } \\ \text { face area }(\mathrm{cm} / \mathrm{s})\end{array} \\ M(a, b, z) & \begin{array}{l}\text { hypergeometric function }\left[=_{1} F_{1}(a ; b ; z)\right] \\ \text { average feed velocity at the inlet }(\mathrm{cm} / \mathrm{s})\end{array} \\ \langle u\rangle & \begin{array}{l}\text { longitudinal velocity along the endothelial cell surface } \\ (\mathrm{cm} / \mathrm{s})\end{array} \\ v_{x} & \begin{array}{l}\text { radial velocity component towards the endothelial cell } \\ \text { surface }(\mathrm{cm} / \mathrm{s})\end{array} \\ v_{y} & \begin{array}{l}\text { component of dimensionless concentration }(c) \text { as a } \\ \text { function of } \xi \text { alone } \\ \text { distance along } x \text {-axis }(\mathrm{cm})\end{array} \\ x(\xi) & \begin{array}{l}\text { component of dimensionless concentration }(c) \text { as a } \\ \text { function of } \gamma \text { alone } \\ \text { distance along } y \text {-axis }(\mathrm{cm})\end{array} \\ y(\gamma) & \text { parameter used in analytical solution }\left[=\lambda\left(\gamma-\frac{1}{2}\right)^{2}\right]\end{array}$

\section{Greek}

$\alpha, \beta \quad$ function defined in terms of Kummer's function

$\lambda_{n}^{2} \quad$ eigenvalues

$\phi^{2} \quad$ thiele modulus $(k h / D)(-)$

$\gamma$ dimensionless radial distance in rectangular duct $(y / h)$

$\mu \quad$ viscosity (cp)

$\tau \quad$ shear stress (dynes $/ \mathrm{cm})$

$\xi \quad$ axial distance $\left(D x /\langle u\rangle h^{2}\right)$ (dimensionless)

\section{Subscript}

$n$ indicating parameter based on $n$th eigenvalue

\section{References}

[1] Pappenheimer, J.R., "Passage of molecules through capillary wall", Physiol. Rev., 33 (1953), 387-423.

[2] Landis, E.M. and Pappenheimer, J.R., "Exchange of substance through the capillary walls", Handbook of Physiology (Am. Physiol. Soc., Washington), Section 2: Circulation, Vol. II (1963), pp. 961-1034. 
[3] Stemerman, M.B., Baumgartner, H.R. and Spaet, T.H., "The subendothelial microfibril and platelet adhesion", Lab. Invest., 24 (1971), 179-186.

[4] Vander, A.J., Sherman, J.H. and Luciano, D.S., Human Physiology: The Mechanisms of Body Function, Tata-McGraw Hill, New Delhi (1981).

[5] Yuan, F., Chien, S. and Weinbaum, S., "A new view of convective-diffusive transport processes in the arterial intima", Trans. ASME (J. Biomech. Eng.), 113 (1991), 314-329.

[6] Furchgott, R.F. and Vanhoutte, P.M., "Endothelium derived relaxing and contracting factors", FASEB J., 3 (1989), 2007-2018.

[7] Hong, S.L., McLaughlin, N.J., Tzeng, C. and Patton, G., "Prostacyclin synthesis and deacylation of phospholipids in human endothelial cells: comparison of thrombin, histamine and ionophore A23187", Thromb. Res., 38 (1985), 1-10.

[8] Hallam, T.J., Pearson, J.D. and Needham, L.A., "Thrombin-stimulated elevation of human endothelial cell cytoplasmic free calcium concentration causes prostacyclin production", Biochem. J., 251 (1988), 243-249.

[9] Pearson, J.D., Carleton, J.S. and Gordon, J.L., "Metabolism of adenine nucleotides by ectoenzymes of vascular endothelial and smooth muscle cells in culture", Biochem. J., 190 (1980), 421-429.

[10] Pearson, J.D. and Gordon, J.L., "Nucleotide metabolism by endothelium", Аnпи. Rev. Physiol., 47 (1985), 617-627.

[11] Nollert, M.U. and McIntire, L.V., "Convective mass transfer effects on the intracellular calcium response of endothelial cells", Trans. ASME (J. Biomech. Eng.), 114 (1992), 321-326.

[12] Mo, M., Eskin, S.G. and Schilling, W.P., "Flow induced changes in calcium signaling of vascular endothelial cells: effect of shear stress and ATP”, Am. J. Physiol., 260 (Heart Circ. Physiol. 29) (1991), H1698-H1707.

[13] Frangos, J.A., Eskin, S.G., McIntire, L.V. and Ives, C.L., "Flow effects on prostacyclin production by culture human endothelial cell”, Science, 227 (1985), 1477-1479.

[14] Diamond, S.L., Eskin, S.G. and McIntire, L.V., "Fluid flow stimulates tissue plasminogen activator secration by cultured human endothelial cells", Science, 243 (1989), 1483-1485.

[15] Grabowski, E.F., Jaffe, E.A. and Weksler, B.B., "Prostacyclin production by cultured endothelial cell monolayers exposed to step increases in shear stress", J. Lab. Clin. Med., 105 (1985), 36-43.

[16] Fry, D.L., "Effect of pressure and stirring on in vitro aortic transmural I-albumin transport", Am. J. Physiol., 260 (Heart Circ. Physiol. 14) (1983), H977-H991.

[17] Olesen, S.P., Clapham, D.E. and Davies, P.F., "Haemodynamic shear stress activates a K current in vascular endothelial cells", Nature, 331 (1988), 168-170.

[18] Davies, P.F., "How do vascular endothelial cells respond to flow?", News Physiol. Sci., 4 (1989), 22-25.

[19] Dull, R.O. and Davies, P.F., "Flow modulation of agonist (ATP) response $\left(\mathrm{Ca}^{++}\right)$ coupling in vascular endothelial cells", Am. J. Physiol., 261(1) (1991), H149-H154.

[20] Friedman, M.H., Bargeron, C.B., Duncan, D.D., Hutchins, G.M. and Mark, F.F., "Effects of arterial compliance and non-Newtonian rheology on correlations between intimal thickness and wall shear", Trans. ASME (J. Biomech. Eng.), 114 (1992), 317-320.

[21] Nerem, R.M., "Vascular fluid mechanics, the arterial wall and atherosclerosis", Trans. ASME (J. Biomech. Eng.), 114 (1992), 274-282.

[22] Okano, M. and Yoshida, Y., "Endothelial cell morphometry of atherosclerotic lesions and flow profiles at aortic bifurcations in cholesterol fed rabbits", Trans. ASME (J. Biomech. Eng.), 114 (1992), 301-308.

[23] Satcher, R.L. Jr., Bussolri, S.R., Gimbrone, M.A. Jr. and Dewey, C.F. Jr., "The distribution of fluid forces on model arterial endothelium using computational fluid dynamics", Trans. ASME (J. Biomech. Eng.), 114 (1992), 309-316. 
[24] Holtz, J., Forstermann, U., Pohl, U., Geisler, M. and Bassenge, E., "Flow dependent endothelium-mediated dilation of epicardial coronary arteries in conscious dogs: Effects of cyclooxygenase inhibition", J. Cardiovasc. Pharmacol., 6 (1984), 1161-1169.

[25] Pohl, U.J., Holtz, R.B. and Bassenge, E., "Crucial role of endothelium in the vasodilator response to increased flow in vivo", Hypertension Dallas, 7 (1986), $37-44$.

[26] Rubanyi, G.M., Romero, J.C. and Vanhoutte, P.M., "Flow induced release of endothelium-derived relxing factor", Am. J. Physiol., 250 (Heart Circ. Physiol. 19) (1986), H1145-H1149.

[27] Kreutzberg, G., Reddington, M. and Zimmermann, H. (eds.), Cellular Biology of Ectoenzymes, Springer-Verlag: Berlin (1986).

[28] Friedli, B., Kent, G. and Olley, P.M., "Inactivation of bradykinin in the pulmonary vascular bed of new born fetal lambs", Circ. Res., 33 (1973), 421-427.

[29] Burnstock, G., Physiological and Regulatory Functions of Adenosine and Adenine Nucleotides, Bassel: Karger (1978), pp. 278-284.

[30] Lieberman, G.E., Lewis, G.P. and Peters, T.J., "A membrane bound enzyme in rabbit aorta capable of inhibiting adenosine-diphosphate induced platelet aggregation", Lancet, 2 (1977), 330-332.

[31] Berridge, M.J., "Inositol trisphosphate and diacylglycerol: two interacting second messengers", Ann. Rev. Biochem., 59 (1987), 159-193.

[32] Berridge, M.J. and Irvine, R.F., "Inositol phosphates and cell signaling", Nature, 341 (1989), 197-205.

[33] Jans, D.A. and Hubner, S., "Regulation of protein transport to the nucleus: central role of phosporylation", Physiol. Rev., 76 (1996), 651-685.

[34] Grimsrud, L. and Babb, A.L., "Velocity and concentration profiles for laminar flow of a Newtonian fluid in dialyzer", Chem. Eng. Prog. Symp. Ser., 62(66) (1966), $19-31$.

[35] Colton, C.K., Smith, K.A., Stroeve, P. and Merril, E.W., "Laminar flow mass transfer in a flat duct with permeable walls", AIChE J., 17 (1971), 773-780.

[36] Coade, S.B. and Pearson, J.D., "Metabolism of adenine nucleotides in human blood", Circulation Research, 65(3) (1989), 531-537.

[37] Berman, A.S., "Laminar flow in channels with porous walls", J. Appl. Phys., 24 (1953), 1232-1235.

[38] Kozinski, A.A., Schmidt, F.P. and Lightfoot, E.N., "Velocity profile in porous walled duct", Ind. Eng. Chem. Fund., 9 (1970), 502-505.

[39] Kleinstreuer, C. and Paller, M.S., "Laminar dilute suspension flow in plate-andframe ultrafiltration units", $A I C h E$ J., 29 (1983), 529-533.

[40] Kumar, V., "Mathematical modelling and computer simulation of membrane processes involving synthetic and natural membranes", Ph.D. Thesis, Dept. of Chem. Eng., Banaras Hindu University, Varanasi (1995).

[41] Slater, L.J., Confluent Hypergeometric Functions, The Synodics of the Cambridge University Press (1960).

\section{APPENDIX}

To solve Eq. (6) along with boundary conditions (7) by the method of separation of variables it is assumed that

$$
c=X(\xi) \cdot Y(\gamma) .
$$


Substituting in Eq. (6) we get two simultaneous differential equations

$$
\frac{\mathrm{d}^{2} Y}{\mathrm{~d} \gamma^{2}}+\lambda^{2}\left(\gamma-\gamma^{2}\right) Y=0
$$

and

$$
\frac{\mathrm{d} X}{\mathrm{~d} \xi}=-\frac{\lambda^{2} X}{6}
$$

The solution of Eq. (A.3) is

$$
X=B \exp \left(-\lambda^{2} \xi / 6\right)
$$

whereas Eq. (A.2) can be transformed to the confluent hypergeometric equation

$$
z \frac{\mathrm{d}^{2} \omega}{\mathrm{d} z^{2}}+(b-z) \frac{\mathrm{d} \omega}{\mathrm{d} z}-a \omega=0
$$

by two transformations

$$
\omega=Y \mathrm{e}^{z / 2}
$$

and

$$
z=\lambda\left(\gamma-\frac{1}{2}\right)^{2}
$$

where $a=\left(\frac{1}{4}-\lambda / 16\right)$ and $b=\frac{1}{2}$. Transformations (A.6) and (A.7) were obtained from the standard transformation of the confluent hypergeometric equation [41] for

$$
\omega=\gamma^{A} \mathrm{e}^{f(\gamma)} Y(\gamma)
$$

and

$$
z=K H(\gamma)
$$


which gives the transformed differential equation

$$
\begin{aligned}
Y^{\prime \prime}+ & \left(\frac{2 A}{\gamma}+2 f^{\prime}+\frac{H^{\prime} b}{H}-K H^{\prime}-\frac{H^{\prime \prime}}{H^{\prime}}\right) Y^{\prime} \\
+ & \left\{\left(\frac{H^{\prime} b}{H}-K H^{\prime}-\frac{H^{\prime \prime}}{H^{\prime}}\right)\left(\frac{A}{\gamma}+f^{\prime}\right) \frac{A(A-1)}{\gamma^{2}}\right. \\
& \left.+\frac{2 A f^{\prime}}{\gamma}+f^{\prime \prime}+f^{\prime 2}-\frac{a K H^{\prime 2}}{H}\right\} Y=0,
\end{aligned}
$$

where primes denote differentiation with respect to $\gamma$.

Equating coefficients of $Y^{\prime}$ to zero and that of $Y$ to $\lambda\left(\gamma-\gamma^{2}\right)$ in Eq. (A.10) with suitable choice of $A, K, H(\gamma)$ and $f(\gamma)$, i.e.,

$$
\begin{gathered}
A=0, \\
K=\lambda, \\
H(\gamma)=\left(\gamma-\frac{1}{2}\right)^{2}
\end{gathered}
$$

and

$$
f(\gamma)=z / 2=\lambda / 2\left(\gamma-\frac{1}{2}\right)^{2}
$$

Eq. (A.10) reduces to Eq. (A.2).

Equation (A.5) is the well known confluent hypergeometric equation (Kummer's equation) and its solution is

$$
\omega=K_{1} M(a, b, z)+K_{2} \sqrt{z} M(1+a-b, 2-b, z)
$$

and the confluent hypergeometric function

$$
M(a, b, x) \equiv{ }_{1} F_{1}(a ; b ; x)=\sum_{n=0}^{\infty} \frac{(a)_{n}}{(b)_{n} n !} x^{n},
$$

where $(a)_{n}=a(a+1)(a+2) \cdots(a+n-1)$ and $(a)_{0}=1$.

For B.C. I and Eq. (A.15) we have at $\gamma=1$ (i.e., $\sqrt{z}=+\sqrt{\lambda} / 2$ )

$$
K_{1} \bar{\alpha}+K_{2} \bar{\beta}=0,
$$


where

$$
\bar{\alpha}=2 a \lambda M\left(a+1, \frac{3}{2}, \lambda / 4\right)-\left(\phi^{2}-\lambda / 2\right) M\left(a, \frac{1}{2}, \lambda / 4\right)
$$

and

$$
\begin{aligned}
\bar{\beta}= & \left(\frac{\lambda}{\sqrt{\lambda}}-\frac{\sqrt{\lambda}}{2}\left(\phi^{2}-\lambda / 2\right)\right) M\left(a+\frac{1}{2}, \frac{3}{2}, \lambda / 4\right) \\
& +\frac{\lambda \sqrt{\lambda}(2 a+1)}{6} M\left(a+\frac{3}{2}, \frac{5}{2}, \lambda / 4\right) .
\end{aligned}
$$

Similarly from B.C. II and Eq. (A.15) we have at $\gamma=0$ (i.e., $\sqrt{z}=-\sqrt{\lambda} / 2)$

$$
K_{1} \alpha+K_{2} \beta=0
$$

where

$$
\alpha=2 a M\left(a+1, \frac{3}{2}, \lambda / 4\right)-\frac{1}{2} M\left(a, \frac{1}{2}, \lambda / 4\right)
$$

and

$$
\begin{aligned}
\beta= & \left(\frac{1}{\sqrt{\lambda}}-\frac{\sqrt{\lambda}}{4}\right) M\left(a+\frac{1}{2}, \frac{3}{2}, \lambda / 4\right) \\
& +\frac{\sqrt{\lambda}(2 a+1)}{6} M\left(a+\frac{3}{2}, \frac{5}{2}, \lambda / 4\right) .
\end{aligned}
$$

Thus from Eq. (A.17) and (A.18) we get the characteristic equation

$$
\alpha \cdot \bar{\beta}-\beta \cdot \bar{\alpha}=0 \text {. }
$$

Roots of the characteristic equation give eigenvalues $\lambda_{n}$. As many as 75 eigenvalues for different $\phi^{2}$ were calculated. A few of them at each $\phi^{2}$ are given in Table AI and a detailed tabular listing may be obtained from authors.

From orthogonality condition [11] we have

$$
A_{n}=\frac{\int_{0}^{1}\left(\gamma-\gamma^{2}\right) Y_{n} \mathrm{~d} \gamma}{\int_{0}^{1}\left(\gamma-\gamma^{2}\right) Y_{n}^{2} \mathrm{~d} \gamma}
$$




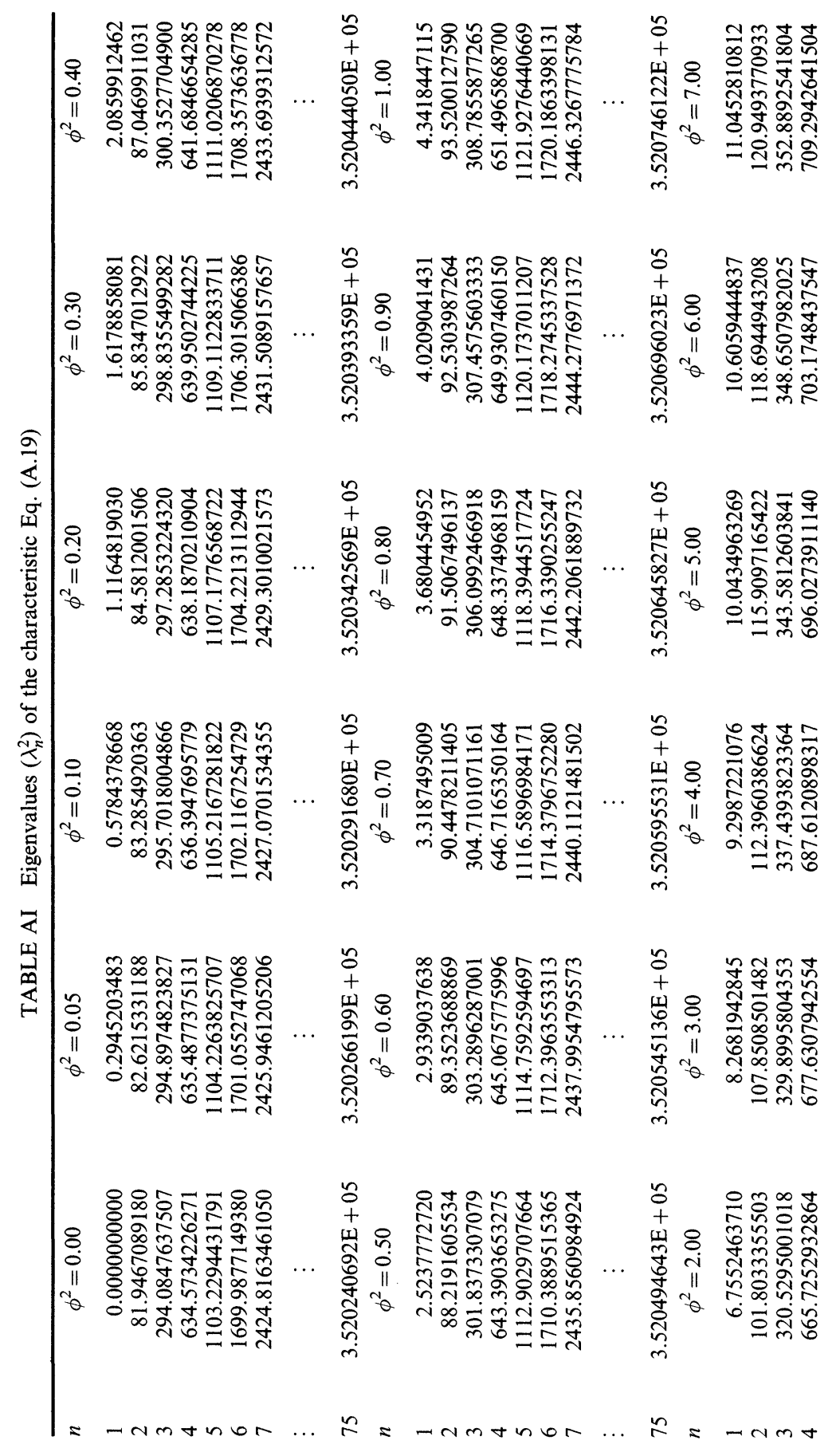



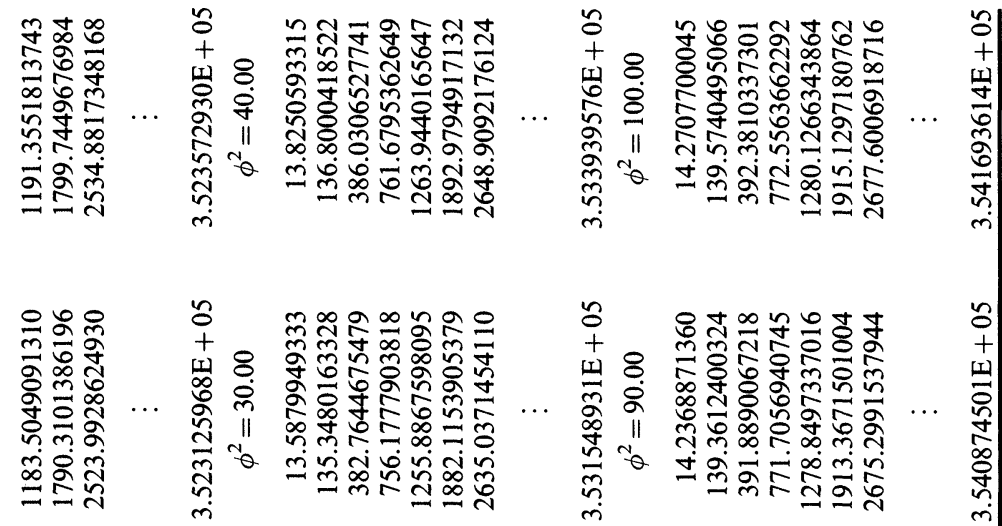

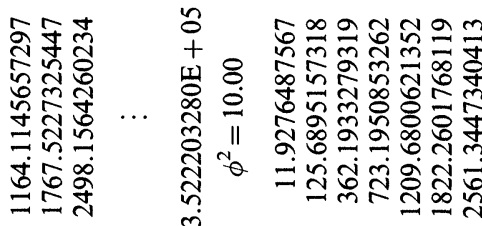
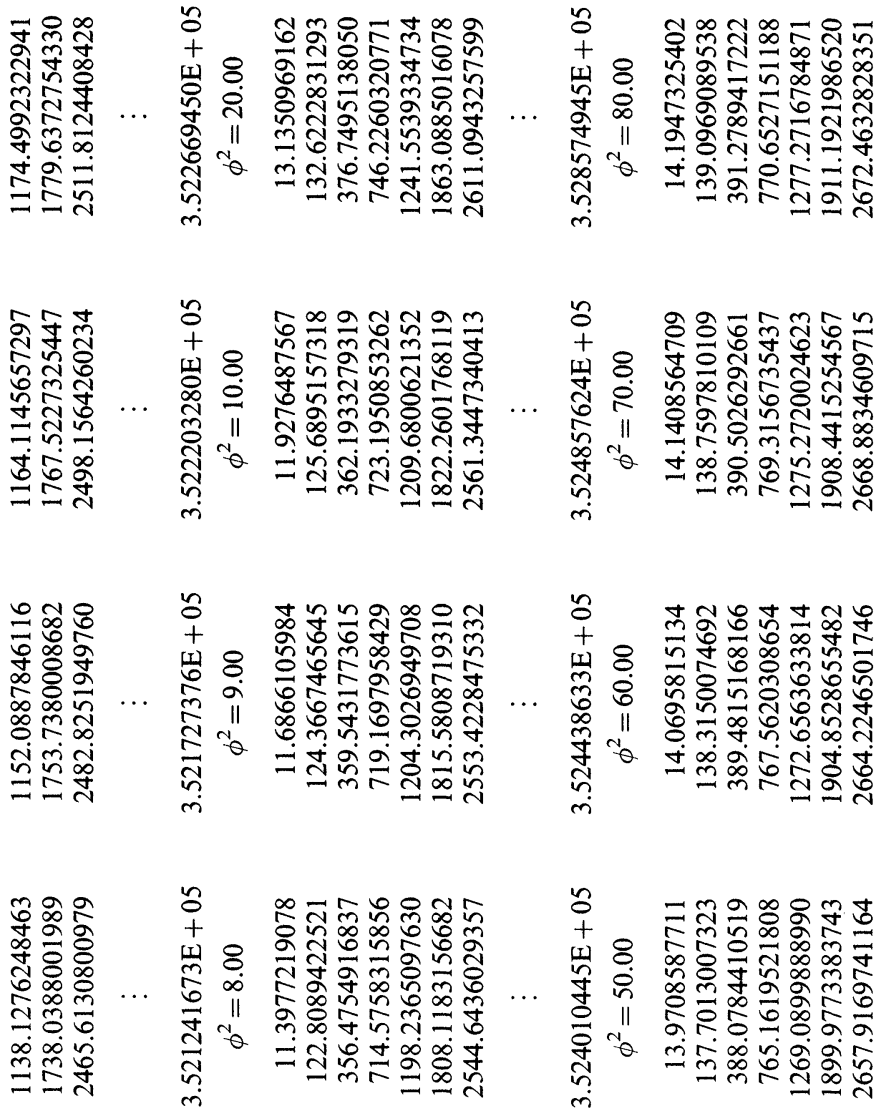

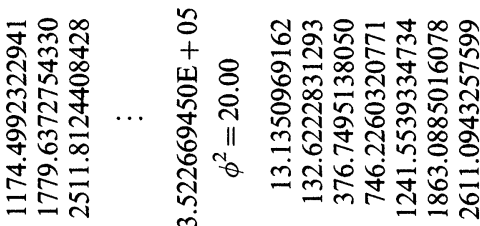

nor... $\cong=$ - rmanor

$n=-\operatorname{rotanon}$ 
Also, since Eq. (A.2) is valid for all $\lambda_{n}$, therefore

$$
\frac{\mathrm{d}^{2} Y_{n}}{\mathrm{~d} \gamma^{2}}+\lambda_{n}^{2}\left(\gamma-\gamma^{2}\right) Y_{n}=0
$$

Integrating Eq. (A.21) within the limit 0 to 1 and using B.C. I and II we get

$$
\int_{0}^{1}\left(\gamma-\gamma^{2}\right) Y_{n} \mathrm{~d} \gamma=\frac{1}{\lambda_{n}^{2}} \phi^{2} \cdot Y_{n}(1)
$$

Again multiplying Eq. (A.21) by $Y_{n}$ and integrating we get

$$
\int_{0}^{1}\left(\gamma-\gamma^{2}\right) Y_{n}^{2} \mathrm{~d} \gamma=\frac{1}{\lambda_{n}^{2}}\left[\phi^{2} Y_{n}^{2}(1)+\int_{0}^{1}\left(\frac{\mathrm{d} Y_{n}}{\mathrm{~d} \gamma}\right)^{2} \mathrm{~d} \gamma\right]
$$

Thus from Eq. (A.20), (A.22) and (A.23) we get

$$
A_{n}=\frac{\phi^{2} Y_{n}(1)}{\phi^{2} Y_{n}^{2}(1)+\int_{0}^{1}\left(\mathrm{~d} Y_{n} / \mathrm{d} \gamma\right)^{2} \mathrm{~d} \gamma}
$$

Also we have

$$
\omega=K_{1} M\left(a, \frac{1}{2}, z\right)+K_{2} \sqrt{z} M\left(a+\frac{1}{2}, \frac{3}{2}, z\right) \quad \text { and } \quad K_{2}=-K_{1}(\alpha / \beta) .
$$

Thus the eigenfunction becomes

$$
Y_{n}=\mathrm{e}^{-z_{n} / 2}\left[M\left(a_{n}, \frac{1}{2}, z_{n}\right)-\frac{\sqrt{z}_{n} \alpha_{n}}{\beta_{n}} M\left(a_{n}+\frac{1}{2}, \frac{3}{2}, z_{n}\right)\right] .
$$

Therefore, at $\gamma=1$, i.e. at $\mathrm{z}_{n}=\lambda_{n} / 4$ (and $\sqrt{z}_{n}=\sqrt{\lambda}_{n} / 2$ ), Eq. (A.25) becomes

$$
Y_{n}(1)=\mathrm{e}^{-\lambda_{n} / 8}\left[M\left(a_{n}, \frac{1}{2}, \lambda_{n} / 4\right)-\frac{\sqrt{\lambda}_{n} \alpha_{n}}{2 \beta_{n}} M\left(a_{n}+\frac{1}{2}, \frac{3}{2}, \lambda_{n} / 4\right)\right] .
$$


Differentiating Eq. (A.25) with respect to $\gamma$ we get

$$
\begin{gathered}
\frac{\mathrm{d} Y_{n}}{\mathrm{~d} \gamma}=\mathrm{e}^{-z_{n} / 2}\left[2 \lambda_{n}\left(\gamma-\frac{1}{2}\right)\left\{2 a_{n} M\left(a_{n}+1, \frac{3}{2}, z_{n}\right)-\frac{1}{2} M\left(a_{n}, \frac{1}{2}, z_{n}\right)\right\}\right. \\
-\frac{\sqrt{\lambda}_{n} \alpha_{n}}{\beta_{n}}\left\{\left(1-z_{n}\right) M\left(a_{n}+\frac{1}{2}, \frac{3}{2}, z_{n}\right)\right. \\
\left.\left.\quad+\frac{\left(2 z_{n}\right)\left(2 a_{n}+1\right)}{3} M\left(a_{n}+\frac{3}{2}, \frac{5}{2}, z_{n}\right)\right\}\right]
\end{gathered}
$$

It is difficult to integrate square of the right-hand side of Eq. (A.27) analytically. To evaluate $A$ using equation (A.24), Eq. (A.27) was integrated numerically by Simpson's $\frac{1}{3}$-rule. 


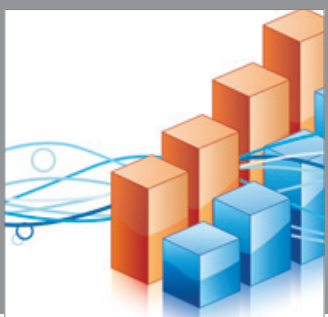

Advances in

Operations Research

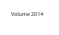

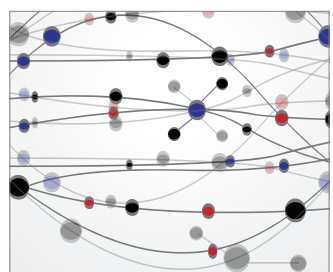

\section{The Scientific} World Journal
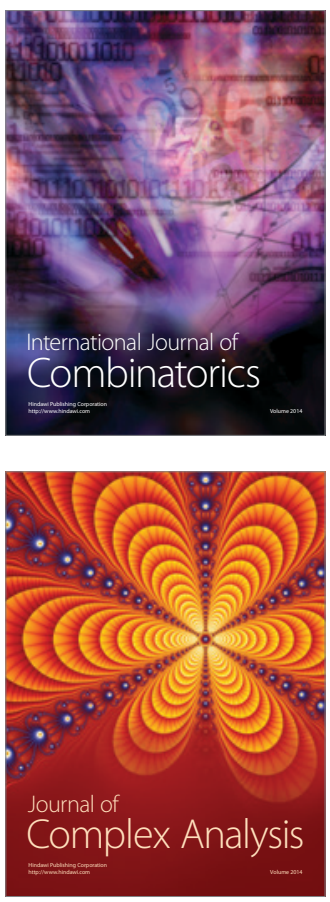

International Journal of

Mathematics and

Mathematical

Sciences
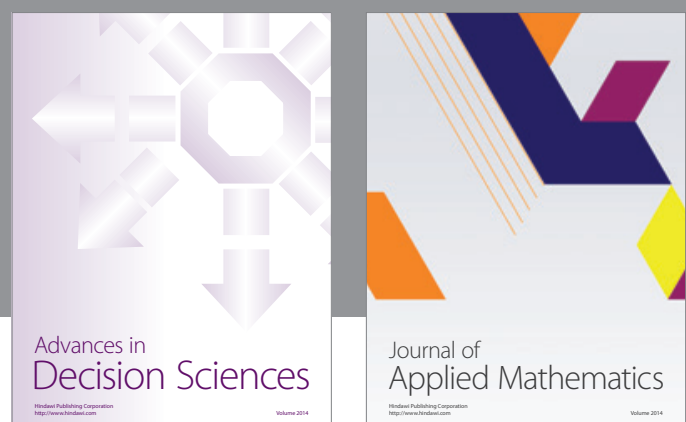

Journal of

Applied Mathematics
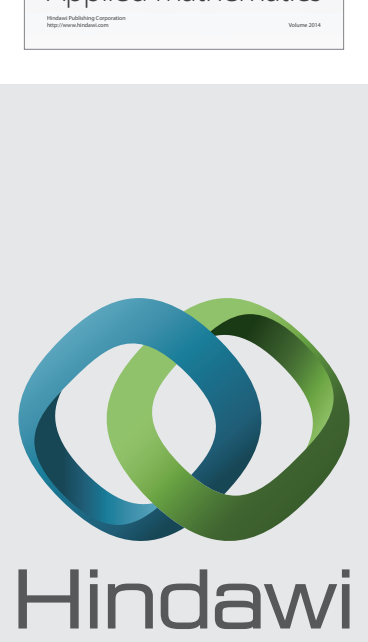

Submit your manuscripts at http://www.hindawi.com
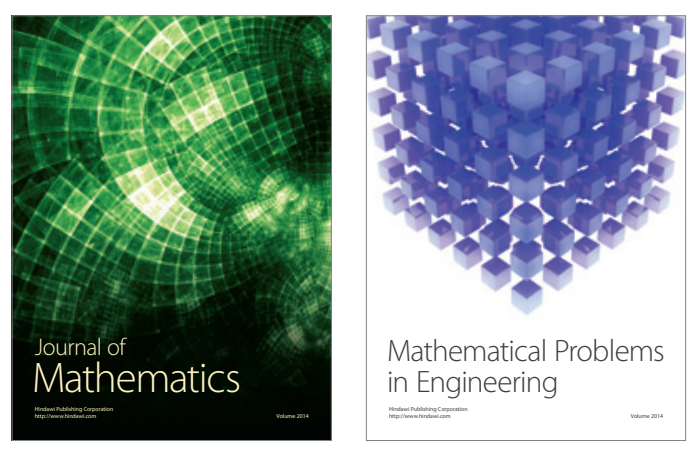

Mathematical Problems in Engineering
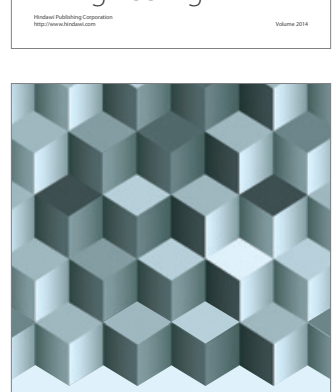

Journal of

Function Spaces
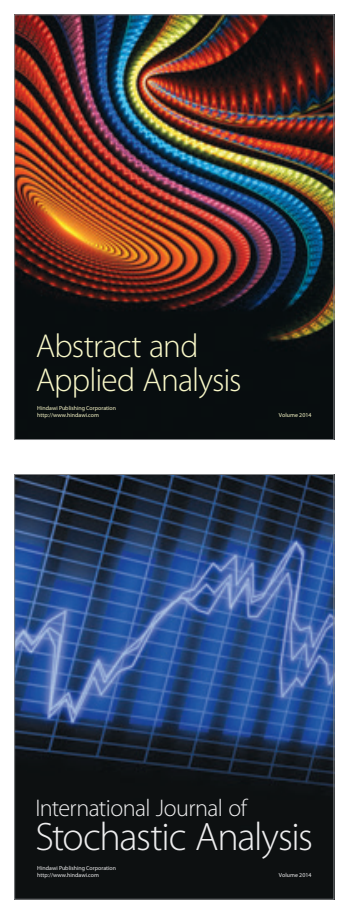

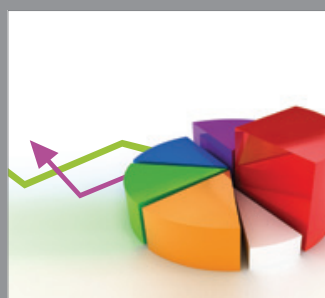

ournal of

Probability and Statistics

Promensencen
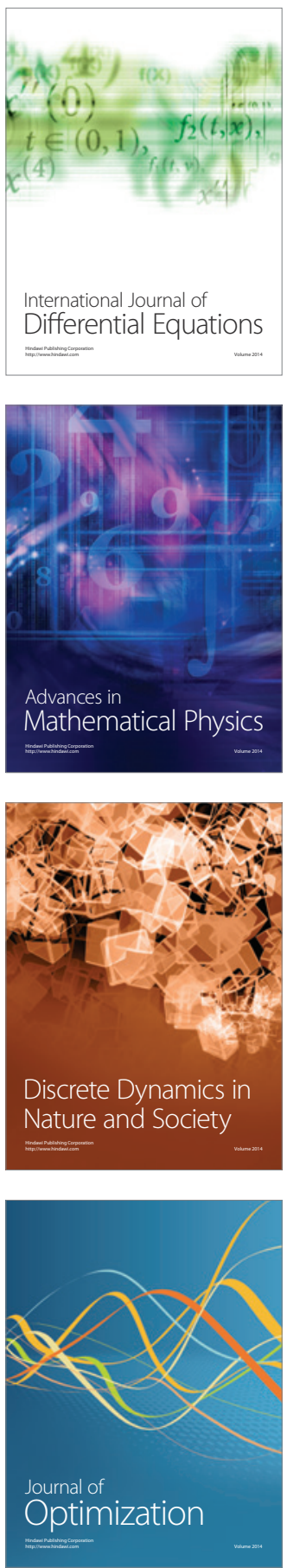